African Crop Science Journal by African Crop Science Society is licensed under a Creative Commons Attribution 3.0 Uganda License. Based on a work at www.ajol.info/ and www.bioline.org.br/cs

DOI: https://dx.doi.org/10.4314/acsj.v28i1.21S

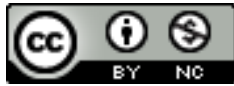

\title{
ANALYSE DE LA GOUVERNANCE DES PETITES ET MOYENNES ENTREPRISES FAMILIALES DE LA VILLE DE BUKAVU EN RÉPUBLIQUE DÉMOCRATIQUE DU CONGO
}

\author{
A. MMENGE ${ }^{1,2}$, B. BINWA ${ }^{1,2}$ et D. GAHUNGU ${ }^{1,2}$ \\ ${ }^{1}$ Faculté des sciences économiques et de gestion, Université du Burundi \\ ${ }^{2}$ Centre Universitaire de Recherche pour le Développement Economique et Social, \\ Université du Burundi \\ Auteur correspondant : adolphemmenge@gmail.com
}

\section{RÉSUMÉ}

Les PME évoluent dans un environnement en perpétuelle évolution, celui-ci oblige les dirigeants de mettre en œuvre des plans d'actions susceptibles d'orienter les actions vers les objectifs visés, de manière à être compétitif et créer de la richesse. L'objectif decette étude était d'analyser et mesurer la gouvernance des PMEF de la ville de Bukavu en République Démocratique du Congo. L'échelle utilisée montre les bonnes propriétés psychométriques $\left(\mathrm{X}^{2}=19.42 ; \mathrm{P}=0.6766 ; \mathrm{X}^{2} / \mathrm{ddl}=0.84 \mathrm{CFI}=0.99\right.$; $\mathrm{IFI}=0.99 ; \mathrm{RMSEA}=0.000 ; \mathrm{NFI}=0.92 ; \mathrm{GFI}=0.97$ et $\mathrm{AGFI}=0.94)$.Cette étude a analysé les données primaires collectées sur 132PMEFde la ville de Bukavu. Le traitement des données a été obtenu en recourant à l'analyse factorielle exploratoire et confirmatoire et au tri à plat. Les résultats indiquent que la gouvernance est un construit à neuf items regroupés en quatre dimensions (la parcimonie, leparticularisme, la personnification et le système de gestion)qui expliquent 68.394 pourcent de la variance totale. Ils montrent que les validités convergente et discriminante sont assurées $(\mathrm{CR}=0.826$ et $\mathrm{AVE}=0.6$; et AVE supérieure au coefficient de corrélation partielle entre les variables). Ils révèlent aussi que les PMEF disposent les avis partagés sur l'importance de la gouvernance et leurs dirigeants disposent la difficulté de mettre en place une bonne gouvernance.

Mots Clés : création de la richesse, PMEF, parcimonie, système de gestion, personnification, particularisme

\begin{abstract}
Small and Medium Enterprises (SME) exploit the environment in a perpetual evolution. This aspect obliges the Managers to implement action plans to direct the exploitation according to the fixed aims, in order to become competitive and create richness. The objective of this study was to analyse and measure the family SME gouvernance of Bukavu in Democratic Republic of Congo. The scale used for measuring family SME gouvernance shows the good psychometric properties $\left(\mathrm{X}^{2}=19.42 ; \mathrm{P}=0.6766\right.$; $\left.\mathrm{X}^{2} / \mathrm{df}=0.84 \mathrm{CFI}=0.99 ; \mathrm{IFI}=0.99 ; \mathrm{RMSEA}=0.000 ; \mathrm{NFI}=0.92 ; \mathrm{GFI}=0.97 \mathrm{et} \mathrm{AGFI}=0.94\right)$. This study analysed the primary data collected on $132 \mathrm{PMEF}$ of Bukavu. The data processing was obtained through the
\end{abstract}


exploratory and confirmatory factor analysis. The results indicate that the gouvernance is a construct with nine items gathered in four dimensions (parsimony, particularism, the personification and the system of management) which explain 68.394 percent of the total variance. They show that the convergent and discriminating validities are assured $(\mathrm{CR}=0.828$; $\mathrm{AVE}=0.6$ and AVE higher than the partial coefficient of correlation between the variables). They also reveal that the family SME managers lay out the divided opinions on the importance of the gouvernance and have the difficulty in setting up a good gouvernance.

Key Words: Richness, family SME, parsimony, particularism, personification

\section{INTRODUCTION}

Les Petites et Moyennes Entreprises (PMEs) évoluent dans un environnement en perpétuelle évolution, celui-ci oblige les dirigeants de mettre en œuvre des plans d'actions susceptibles d'orienter les actions vers les objectifs visés, de manière à être compétitif et créer de la richesse (Geniaux et Bonnardel, 2001). Cet aspect prouve la nécessité de la mise place d'un système de contrôle et d'orientation d'action pour assurer la flexibilité des PME (Kolsi et Ghorbel, 2011). Ce système par lequel les entreprises sont dirigées et contrôlées est appelé gouvernance (Yassine, 2013). Elle oriente les décisions stratégiques et opérationnelles de l'entreprise engageant les ressources financières du propriétaire-dirigeant dans l'objectif de la création de la richesse (Cerrada et Janssen, 2006). Adapté aux PMEF, les raisonnements ci-dessus se justifient par le fait que plus le dirigeant propriétaire obtient la confiance des autres membres de la famille pour diriger le patrimoine commun (Bughin et al., 2010),maintenir la réputation et l'image de la famille pour assurer la performance de l'entité et ainsi la léguer ou la transmettre aux générations futures (Bauweraerts et Colot, 2015).Le dirigeant a l'obligation d'assurer la convergence des intérêts de tous les membres vers les objectifs préalablement établis pour assurer l'indépendance et la survie de l'entreprise à travers une bonne adéquation de la relation famille- entreprise - propriété. Pour y arriver, le dirigeant doitadopter une bonne gouvernance (Hirigoyen, 2007).

Les entreprises familiales jouent un rôle considérable dans l'économie de la plupart des pays. Elles représentent entre 50 et $90 \%$ du produit intérieur brut de toutes les économies de marché (Kenyon-Rouvinez et Ward, 2004). Cependant, dans la pratique on constate que la plupart des PME sont des entreprises familiales (Il sied de noter que toute entreprise familiale n'est pas une PME, il en existe de grande entreprise) (Comblé et Colot, 2006) dont la plupart disposent en Afrique d'un statut d'entreprise individuelle. Elles représentent un pourcentage important dans le total des entreprises, jusqu'à plus des deux tiers des entreprises dans les pays occidentaux et la quasi-totalité des PME en Afrique Subsaharienne (Morck et Yeung, 2003 ; Adolphe, 2018 ; Landier et al., 2004). Elles représentent $82 \%$ des PME en Allemagne, $83,2 \%$ en Autriche, $81,33 \%$ en Belgique (Bauweraerts et Colot, 2015), plus de $70 \%$ en Malaisie (Claessens et al., 2000) et $79 \%$ en République Démocratique du Congo (Adolphe, 2018). Elles emploient plus de $75 \%$ de mains d'œuvre dans le monde, créent plus de $86 \%$ de nouveaux emplois aux Etats unis (Ramadani et al., 2015) et plus de $80 \%$ en RDC. Elle est une source de valorisation des métiers et des compétences transmise de génération en génération (Attouch et Amria, 2016).

Dans le contexte de la RDC, le tissu économique est essentiellement constitué des PME. Un total de $80,7 \%$ des dirigeants des PME sont propriétaires de la PME (Adolphe, 2018); 72,7\% des PME sont familiales (Banque Mondiale, 2014) et dont le propriétaire dirigeant constitue le principal stakeholder (Ngongang, 2005). Le propriétaire-dirigeant s'implique dans tous les aspects de la gestion (St-Pierre et Cadieux, 2011). La PMEF 
constitue la cellule de base pour la relance de l'économie d'un pays du fait qu'elle est disséminée à travers le territoire du pays et surtout qu'elle n'exige pas d'investissement couteux et de transfert massif des devises (Ndalo, 2010). Il est pragmatique qu'à l'heure actuelle, on ne peut pas se soustraire du rôle important des PME dans le processus de développement d'un pays car celles-ci constituent non seulement une économie de proximité mais aussi et surtout le poumon de développement(Kamba-Kibatshi, 2016).

L'analyse des PMEF de la ville de Bukavu permet de relever les constats suivants : (i) les pratiques de gestion dans les PMEF sont implicite, opaques et informelle (Geniaux et Bonnardel, 2001). 64.4\% des dirigeants des PMEF éprouvent des difficultés de communication financière et de la tenue des outils de gestion ainsi que les besoins d'accompagnement financier (Adolphe, 2018). (ii) la gouvernance de PME est plus ou moins efficace selon le niveau d'asymétrie entre les acteurs impliqués dans la gestion de l'entreprise(Hirigoyen, 2007). (iii) les difficultés d'accès au marché extérieur, le non accès aux crédits, le manque de politique claire en faveur des PME(Ndalo, 2010).(iv)les choix stratégiques dans les PMEF sont influencés par la famille, la propriété, le mode de gouvernance et la perception de la succession par ses dirigeants (Ntambaka, 2015).

Force est de constater, la quasi-totalité des difficultés relevées dans les PMEF proviennent de la gouvernance. Ces constats révèlent que, si la bonne gouvernance est intégrée dans les PMEF, ces dernières peuvent surmonter les obstacles auxquels elles se heurtent.Face à l'importance des PMEF et leur rôle inéluctable, il faut de ce fait examiner leurs gouvernances comme solution aux difficultés auxquelles elles font face.

Le développement des échelles de mesure capables d'évaluer la gouvernance a donné naissance à des études dans les grandes entreprises.Carney,2005 ;Assia,2016 et Bach et al., 2018sont considérés comme les pionniers pour avoir développé l'échelle de mesure de la gouvernance dans le contexte avec marché financier pour les PME en générale. Ces échelles posent des difficultés de reproduire la même structure factorielle si elles sont appliquées dans le contexte comme celui de la RD Congo où le niveau d'éducation financière des dirigeants est relativement bas et suivant la spécificité des PMEF.

Les études qui visent à développer des échelles de mesure de la gouvernance dans les PMEF sont quasi inexistantes. Celles qui existent sont souvent exploratoires et explorent de manière théorique et exploratoire la gouvernance au sein de l'entreprise familiale (Attouch et Amria, 2016) ou encore la gouvernance cognitive (Bughin et al., 2010). Les autres études sont plus générales et portent sur les pratiques de gouvernance dans les PME et son impact sur la performance (André et al., 2006; Yassine, 2013 ; Kamala, 2014 ; Khaldi, 2014 ; Essama et al., 2015) sans vouloir construire une échelle de mesure de la gouvernance fiable et valide propre aux PMEF dans un contexte sans marché financier comme celui de la ville de Bukavu.

La présente étude voulait combler ce gap et poursuivait les objectifs complémentaires suivants: (a) Analyser et mesurer la gouvernance des PMEF et discuter les propriétés psychométriques de l'échelle utilisée et (b) identifier les dimensions et les attributs que les dirigeants valorisent au sein des PMEF ainsi que les poids qu'ils leurs accordent (Tableau 1).

\section{Revue de la littérature}

Définition et opérationnalisation de la gouvernance. La gouvernance a déjà fait l'objet de plusieurs définitions (O'grady, 2002 ; Cerrada et Janssen, 2006; Colot et Mapisanas, 2007 ; Djimnadjingar, 2012 ; Yassine, 2013 ; Kolsi et Ghorbel, 2011 ; Mbenda et Sebigunda, 2015). L'analyse minutieuse des définitions existantes telle qu'elle a été effectuée parMendy et Diop, 2018 ; Assia, 2016 ; Chareau et Schatt, 2005, fait ressortir les caractéristiques principales 
TABLEAU 1. Descriptions des attributs par dimensions retenues de la gouvernance des PMEF

\begin{tabular}{|c|c|c|}
\hline Considération concept & Dimensions et auteurs & Description des attributs \\
\hline \multirow[t]{2}{*}{$\begin{array}{l}\text { Gouvernance entrepreneuriale/ familiale } \\
\text { (mécanisme interne d'allocation, de } \\
\text { distribution, d'exercice et de contrôle } \\
\text { des richesses) }\end{array}$} & $\begin{array}{l}\text { Parcimonie : } \\
\text { Kolsi et Ghorbel, 2011; Cerrada et Janssen, } 2006 \text {; } \\
\text { Essama et al. 2015; Assia, 2016Carney, 2005; } \\
\text { Mendy et Diop, } 2018\end{array}$ & $\begin{array}{l}\text { Oriente les décisions stratégiques de l'entreprise } \\
\text { et de la famille; } \\
\text { Minimise le risque d'erreur et asymétrie d'information } \\
\text { financière ; } \\
\text { Oriente les décisions opérationnelles de l'entreprise ; } \\
\text { Améliore la maîtrise de la situation financière et } \\
\text { l'évaluation de la rentabilité ; } \\
\text { Réduire la probabilité d'opportunisme et accroit les } \\
\text { choix raisonnés ; } \\
\text { Précise les limites de pouvoir des dirigeants de la } \\
\text { PMEF }\end{array}$ \\
\hline & $\begin{array}{l}\text { Personnification : } \\
\text { Krafft et al., 2010; Cerrada et Janssen, } 2006 \text {; } \\
\text { Essama et al. } 2015 \text {; Carney, 2005; } \\
\text { Mendy et Diop, } 2018\end{array}$ & $\begin{array}{l}\text { Apprends aux dirigeants à suivre de manière régulière } \\
\text { les objectifs visés et les communiqués aux employés ; } \\
\text { Permet aux dirigeants de faire preuve d'intégrité et } \\
\text { d'équité ; } \\
\text { Renforce la coopération et développe l'esprit } \\
\text { d'équipe ; } \\
\text { Permet aux dirigeants d'être efficace et d'assurer une } \\
\text { bonne démarche objectif - variable - action ; } \\
\text { Permet le respect des procédures administratifs } \\
\text { suivant les statuts et les orientations du conseil de la } \\
\text { famille ; } \\
\text { Accroit le degré de surveillance du dirigeant sur les } \\
\text { respects de normes }\end{array}$ \\
\hline
\end{tabular}


TABLEAU 1. Contd.

\begin{tabular}{|c|c|c|}
\hline Considération concept & Dimensions et auteurs & Description des attributs \\
\hline & $\begin{array}{l}\text { Particularisme : } \\
\text { Cerrada et Janssen, } 2006 \text {;Kolsi et Ghorbel, 2011; } \\
\text { Khaldi, } 2014 \text {; Kamala, } 2014 \text {; Carney, 2005; } \\
\text { Mendy et Diop, } 2018\end{array}$ & $\begin{array}{l}\text { Permet aux gestionnaires d'identifier les nouveaux } \\
\text { secteurs de marchés ; } \\
\text { Permet d'identifier les nouvelles opportunités } \\
\text { d'affaire ; } \\
\text { Permet de réduire les risques de perte et d'identifier } \\
\text { les activités rentables et les créneaux porteurs ; } \\
\text { Renforce les suivis quotidiens des activités de } \\
\text { l'entreprise ; } \\
\text { Permet d'affecter la responsabilité et de corriger les } \\
\text { erreurs. }\end{array}$ \\
\hline \multirow[t]{2}{*}{$\begin{array}{l}\text { Gouvernance d'alliance } \\
\text { (Séparation propriété, pouvoir et } \\
\text { réseau de relation) }\end{array}$} & $\begin{array}{l}\text { Système de gestion : } \\
\text { Essama et al., 2015;André et al., 2006; } \\
\text { Yassine, } 2013 \text {; Khaldi, } 2014\end{array}$ & $\begin{array}{l}\text { Permet de faire de planification stratégiques et } \\
\text { tactiques ; } \\
\text { Renforce les techniques d'évaluation et } \\
\text { d'orientations vers les objectifs visés ; } \\
\text { Facilite le contrôle et orientation des actions ; } \\
\text { Facilite les échanges d'informations entre partenaires } \\
\text { et constitue une source d'apprentissage ; } \\
\text { Veuille aux respects de normes comptables et à la } \\
\text { transmission d'information. }\end{array}$ \\
\hline & $\begin{array}{l}\text { Parties prenantes : } \\
\text { Assia, } 2016 \text {; Cerrada et Janssen, } 2006 \text {; } \\
\text { Hirigoyen, } 2007 \text {; Kamala, } 2014 \text {; } \\
\text { Essama et al., } 2015 \text {; Mendy et Diop, } 2018\end{array}$ & $\begin{array}{l}\text { Améliore la relation avec les partenaires et facilite } \\
\text { l'accès aux ressources; } \\
\text { Améliore les revenus de la PMEF ; } \\
\text { Facilite la couverture des besoins des clients et des } \\
\text { membres de la famille, } \\
\text { Améliore la capital social et la relation entre les } \\
\text { membres de la famille; } \\
\text { Veuille à la rémunération des propriétaires et à la } \\
\text { réputation de la famille. }\end{array}$ \\
\hline
\end{tabular}


ci- après de la gouvernance de l'entreprise : (1) elle est l'ensemble des règles (configurations de la propriété); (2) elle est constituée du réseau de relation (entre les parties prenantes) ; (3) structure et mécanisme d'allocation, de distribution, d'exercice et de contrôle de pouvoir (management de management) ; (4) assure l'autonomie et la création de la valeur au profit des dirigeants c'est-à-dire régule les actions des dirigeants pour une création optimale de la richesse.

Dans cette étude la gouvernance des PMEF est abordée au niveau de mécanismes internes en mettant en exergue la manière dont les PME sont dirigées et contrôlées d'une part, et dont elles créent et répartissent la richesse entre les parties prenantes d'autre part (Mendy et Diop, 2018). A la lumière de Cerrada et Jansen, 2006 ; Essama et al., 2015 ;Assia, 2016 ; Bach et al., 2018, la gouvernance est conçue à la fois comme la gouvernance d'alliance et la gouvernance entrepreneuriale. Elle est analysée en fonction de différents mécanismes impliqués dans le management de l'entreprise pour créer de la valeur aux différents intervenants (amélioration de la rentabilité, incitation de la communication, amélioration des stratégies financières, amélioration de la gestion de relation et de la cohésion entre les intervenants). Tous ces éléments se rapportent au mécanisme interne décrit comme un ensemble des stratégies mis au point par les dirigeants pour orienter les actions au sein de l'organisation (Bocquet et Mothe, 2008). Pareille considération laisse présager que les dirigeants des PMEF évaluent la gouvernance de leurs PMEF en fonction de la capacité à entrainer la cohésion mais aussi de leur système de gestion et de la parcimonie(Aliyua et al., 2014).

La gouvernance des PME est mesurée au moyen des attributs spécifiques (Carney, 2005; Mendt et Diop, 2018 ; Caillie et Onana, 2012 ; Bocquet et Mothe, 2008). La gouvernance est considérée comme une variable latente (non observable) approximée au moyen des attributs observables, mesurables et susceptibles d'être regroupés en facteurs pouvant influencer directement la gouvernance globale (Arrègle et Isabelle, 2010; Bughin et al., 2010 ; Mbenda et Sebigunda, 2015 ). En effet, les dirigeants des entreprises sont sensés prendre en compte les aspects spécifiques de la gouvernance comme par exemple la taille du conseil d'administration, le système de gestion, la surveillance et le contrôle pour réguler les actions, la relation entre les parties prenantes, séparation entre propriété, pouvoir et contrôle, délégation de responsabilité entre autres en vue de permettre une évaluation de la performance des différentes ressources de l'organisation comme les humains, les finances et les stratégies de l'organisation (Chareau et Schatt, 2005 ; Kolsi et Ghorbel, 2011 ; Bach et al., 2018 ; Assia, 2016).

Les composants cités ci-dessus s'harmonisent parfaitement avec la vérité des grandes entreprises et certaines PME cotées (Bughin et al., 2010); la réalité et les caractéristiques de PMEF ne permettent pas d'utiliser ces composantes (Kamala, 2014). Dans ce cas, les caractéristiques comme la parcimonie, la personnification, le particularisme, le système de gestion et la liaison entre les parties prenantes internes (Cerrada et Janssen, 2006; Essama et al., 2015), conviennent aisément avec les réalités des PMEF (Carney, 2005).

Pareille opérationnalisation du concept de la gouvernance des PMEF est dictée par les arguments suivants : Premièrement, elle est compatible avec les représentations que les propriétaires dirigeants des PMEF se font en rapport avec la gouvernance de leurs entreprises (Adolphe, 2018). Deuxièmement, le conseil d'administration est souvent absent dans les PMEF souvent remplacé par le conseil de familleet le conseil consultatif (Aliyua et al., 2014 ; Mendy et Diop, 2018) ; elle offre donc la possibilité de tenir compte de la spécificité des PMEF. Troisièmement, une analyse au niveau des attributs permet de les classer en ordre d'importance en précisant lesquels 
disposent plus d'impact sur la gouvernance globale (Cerrada et Janssen, 2006; Kolsi et Ghorbel, 2011 ; Khaldi, 2014). Quatrièmement, elle fournit la possibilité d'apprécier la relation propriété-famille-entreprise et permet de traduire les résultats en des implications managériales directes en termes d'actions. D'où les attributs par dimension comme repris dans le tableauci-dessous :

De ce qui précède, nous postulons que. $\mathrm{H} 1$ : les parties prenantes, la parcimonie, la personnification, le particularisme et le système de gestion seraient les dimensions permettant de mesurer la gouvernance des PMEF de la ville de Bukavu.

Nécessité de l'analyse et de mesure de la gouvernance des PMEF. La gouvernance des entreprises a déjà donné lieu à plusieurs études et échelles de mesuresdans les grandes entreprises (Charreaux, 1998; Brouard etDivito, 2008 ; Djimnadjingar, 2012) ; dans les PME cotées (Aliyua et al., 2014) et dans les PMEF (Bughin et al., 2010). La majorité de ces études sont exploratoires (Charreaux et Schatt, 2005) et analysent les pratiques ainsi que les théories relatives à la gouvernance adaptées aussi bien dans les grandes que dans les petites et moyennes entreprises à travers une étude des cas ou une analyse de contenue (Cerrada et Janssen, 2006; Hirigoyen, 2007 ; Charreaux, 2008; Mendy et Diop, 2018). Les résultats conjugués de ces études montrent que la gouvernance est applicable dans toutes les entreprises mais sa mise en pratique diffère selon les caractères spécifiques, la structure de propriété et le contexte socio culturelle de chaque entreprise(Mendy et Diop, 2018). Les résultats de ces études révèlent aussi que les principes de gouvernance sont très peu développés dans les PMEF (Bocquet et Mothe, 2008) et montrent la nécessité de porter une analyse de la gouvernance des PMEF dans le contexte de la ville de Bukavu en RD Congo. Ces résultats laissent présumer que le niveau de la gouvernance des PMEF est en deçà de la moyenne. L'argument ci-dessus est en moitié justifié par le fait que l'implication de la famille dans le management de l'entreprise augmente le niveau d'interaction sociale susceptible de réduire l'importance accordée aux différentes facettes de la gouvernance. D'autres études mettent en relation la gouvernance et la performance des entreprises (Bughin et al., 2010 ; Premont, 2016; Belkebir et al., 2018; Mendy et Diop, 2018). L'analyse rapide des résultats de ces études révèle que le mécanisme de la gouvernance influence significativement la performance des entreprises. Les mécanismes de la gouvernance adoptés et leurs impacts sur la performance diffèrent selon que le dirigeant est propriétaire ou non d'une part; d'autre part selon que le dirigeant est interne ou externe à la famille.

Les autres études évaluent la gouvernance en l'approximant par les indices (Colot et Mpasinas, 2007 ; Brouard et Divito, 2008 ; Aliyua et al., 2014 ; Khaldi, 2014 ; Mbenda et Sebigunda, 2015 ; Mendy et Diop, 2018). La plupart de ces études ont utilisé la taille de conseil d'administration, la satisfaction des attentes des parties prenantes et le système de management pour évaluer la gouvernance des entreprises y compris les PMEF (Cerrada et Janssen, 2006). Les résultats agrégés de ces études aboutissent à une instabilité de la dimensionnalité de la gouvernance et conseillent aux entreprises d'analyser et mesurer la gouvernance suivant la spécificité de l'entreprise et du contexte (Caillie et Onana, 2012). Par conséquent, toute échelle de mesure a besoin d'une validation continuelle chaque fois qu'elle serait utilisée dans des pays et contextes culturels différents (Hirigoyen, 2007 ; Bughin et al., 2010; Haguma et al., 2019). Quelques études ont tentées de développer les échelles de mesure et mesurer directement la gouvernance des PMEF (Carney, 2005 ; Bughin et al., 2010) dans les Pays développés et en contexte avec marché financier. Ces échelles posent des difficultés d'adaptation au contexte particulier de la ville de Bukavu et prouvent la nécessité de mesurer 
la gouvernance des PMEF selon les attributs adaptés à la réalité et à la culture du contexte de cette étude. Les aspects sus évoqués nous permettent de formuler l'hypothèse suivante : H2 : le niveau de gouvernance des PMEF de la ville de Bukavu serait légèrement supérieur à la moyenne (Ndjambou and Sassine, 2014)

\section{MÉTHODOLOGIE}

Echantillon. La présente étude analyse les données primaires collectées sur les dirigeants des PME de la ville de Bukavu. L'accent placé sur les dirigeants de la ville de Bukavu est dicté par l'accessibilité et la concentration des PME à Bukavu. Selon le rapport de l'Inspection Provinciale des Petites et Moyennes Entreprises et Artisanats du Sud Kivu 2018 (IPMEA/S-K), la population des PME de la ville de Bukavu est de 2.143 entreprisesqui constituent la population cible de cette étude.La présente étude a ciblé 275 dirigeants et a utiliséun questionnaire d'enquête comprenant deux parties. La première partie a repris les questions fermées et ouvertes relatives aux profils des dirigeants et de la PME. La deuxième partie a compris une échelle multi item relative aux différentes dimensions de la gouvernance et de la performance. Une échelle de mesure du type Likert à 5 niveaux allant de (1. Tout à fait d'accord ; 2. D'accord ; 3 . Neutre ; 4. Pas d'accord et 5. Pas du tout d'accord) a été utilisée pour les deux variables. Sur un total de 275 questionnaires, un suivi régulier a permis de récupérer 195 questionnaires, soit un taux de non-réponse de $29.1 \%$. Des questionnaires bien remplis, 132 ont répondus aux caractères des PME familiales et ont été considéré comme individus échantillons dont les résultats ont été généralisés sur toutes les PMEF.

Echelle de mesure de la gouvernance. La gouvernance est une variable latenteou non observables (Memmi, 2002). Elle a été mesurée par le biais des échelles de mesure antérieures inspirées fortement de la littérature (Carney,
2005 ; Essama et al., 2015). L'échelle de mesure utilisée dans cette étude comprend 17 items regroupés en 5 dimensions dont Parcimonieavec 3 items ; Personnification avec 4 items ; Parties prenantesavec 3 items ; Système de gestion avec 4 items et Particularismes avec 3 items. Le processus de purification de l'échelle a permis de retenir 9 items regroupés en 4 dimensions (Parcimonie, personnification, système de gestion et particularisme). Le tableau cidessous donne de plus amples informations sur les items, les dimensions, les preuves de fiabilité et de validité ainsi que les indices d'ajustement se rapportant au construit latent.

Les résultats de l'analyse factorielle exploratoire indiquent les données admettent l'analyse $(\mathrm{KMO}=0.683$, Khi deux $=194.246$; $\mathrm{P}=0.000)$. Ils révèlent que la gouvernance des PMEF est un construit à quatre dimensions qui expliquent 68.394 pourcent de la variance totale. Le test de fiabilité $(\alpha=0.701)$ montre qu'il existe une cohérence interne entre les items et montre que l'échelle est fiable. Les résultats de l'analyse factorielle confirmatoire démontrent des bonnes propriétés psychométriques pour cette échelle $\left(\mathrm{X}^{2}=19.42 ; \mathrm{P}=0.6766 ; \mathrm{X}^{2} / \mathrm{ddl}=0.84 \mathrm{CFI}=0.99\right.$; $\mathrm{IFI}=0.99 ; \quad \mathrm{RMSEA}=0.000 ; \quad \mathrm{NFI}=0.92$; GFI=0.97; AGFI=0.94) (Tableau 2). Les résultats montrent que chaque variable est statistiquement liée à son construit latent auquel elle était supposée être attachée. Toutes les dimensions se rapportant au construit gouvernance présentent un t-value supérieur à 1.96. Ces résultats peuvent être visualisés dans le diagramme ci-après :

L'échelle utilisée a été soumise à l'analyse de fiabilité et de validité des construits (Fig. 1). Le Tableau ci-dessous présente les amples informations:

Les résultats du Tableau ci-dessus révèlent que le construit dispose l'AVE comprise entre 0.4 et 0.7 et prouve que l'AVE se trouve dans une bonne limite (Hanana et Houfaidi, 2016). Les résultats relatifs à la fiabilité sont aussi satisfaisants (CR>0.70) (Bagozzi et Yi, 2012) 
TABLEAU 2. Rapport de l'analyse factorielle exploratoire et confirmatoire $(\mathrm{N}=132)$

\begin{tabular}{|c|c|c|c|c|}
\hline \multirow[t]{2}{*}{ Description des variables } & \multicolumn{2}{|c|}{ Coefficients structurels } & \multirow[t]{2}{*}{$\mathrm{CR}$} & \multirow[t]{2}{*}{ AVE } \\
\hline & $\mathrm{AFE}$ & $\mathrm{AFC}$ & & \\
\hline $\begin{array}{l}\text { GOUVERNANCE }( \pm=0.701 ; \mathrm{KMO}=0.683 ; \mathrm{CFI}=0.99 ; \mathrm{IFI}=0.99 ; \mathrm{RMSEA}=0.000 ; \\
\mathrm{NFI}=0.92 ; \mathrm{GFI}=0.97 ; \mathrm{AGFI}=0.94)\end{array}$ & & & 0.828 & 0.6 \\
\hline \multicolumn{5}{|l|}{ PARCIMONIE } \\
\hline PARCI2 Limite de pouvoir de dirigeant propriétaire & & & 0.67 & 0.51 \\
\hline & 0.816 & 0.52 & & \\
\hline PARCI3Implication de la famille dans la prise de décision & 0.771 & 0.72 & & \\
\hline PERSONIFICATION & & & 0.803 & 0.7 \\
\hline PERSO1 Rencontre avec les employés et conseil de famille & 0.83 & 0.78 & & \\
\hline PERSO2 Communication avec les employés et cohésion famille & 0.8670 .69 & & & \\
\hline PARTICULARISME & & & 0.803 & 0.683 \\
\hline PARTI2 Identification des nouveaux secteurs d'activité & 0.7720 .92 & & & \\
\hline PARTI3 Identification des créneaux porteurs & 0.8830 .55 & & & \\
\hline SYSTEMEDEGESTION & & & 0.62 & 0.4 \\
\hline SYSGE2 Respect des norms et procedures de gestion & 0.7080 .54 & & & \\
\hline SYSGE3Mise en place des outils de contrôle et évaluation & 0.596 & 0.59 & & \\
\hline SYSGE4 Etablissement quotidien des rapports & 0.7620 .42 & & & \\
\hline
\end{tabular}

Comparative Fit Index; IFI = Incremental Fit Index; RMSEA = Root Mean Square Error of Approximation; NFI = Normal Fit Index; GFI = Goodness of Fit Index; AGFI = Adjusted Goodness of Fit Index 


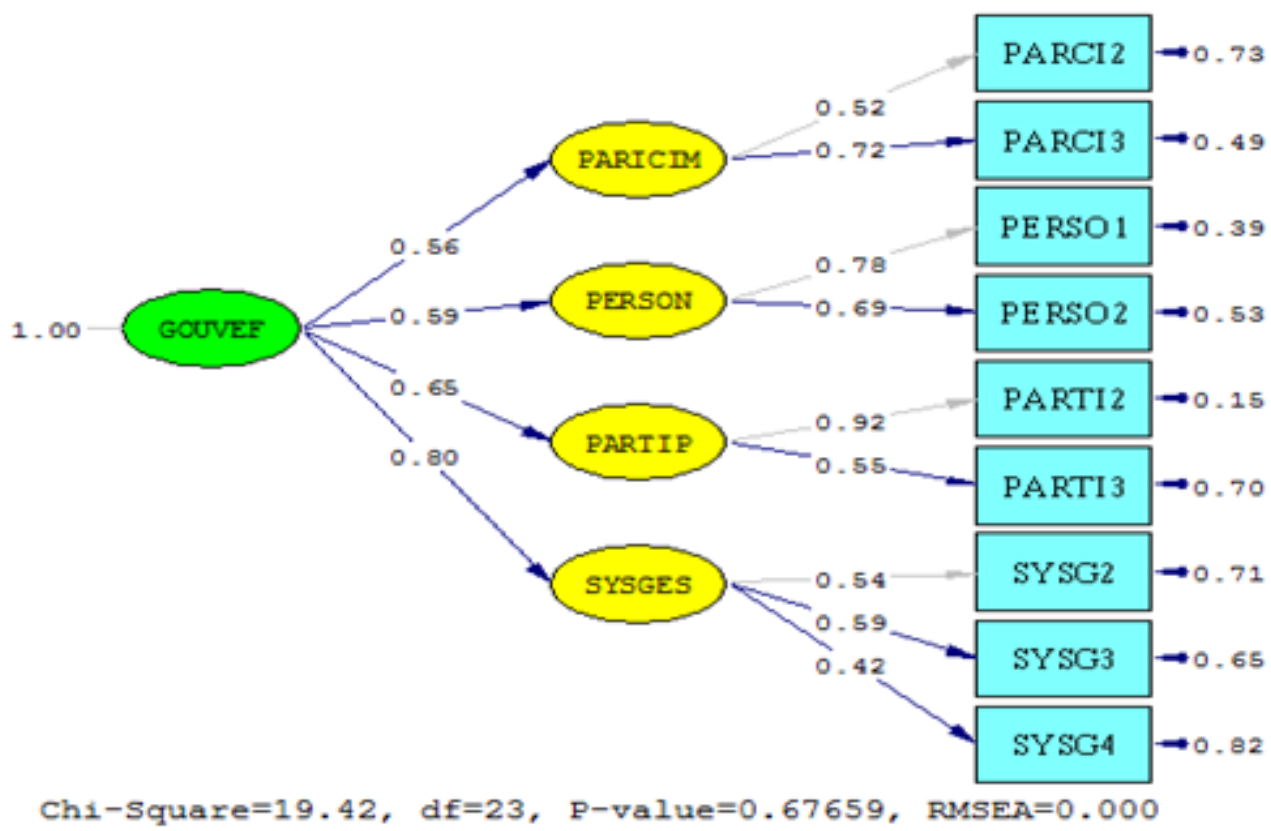

Figure 1. Mesure de la gouvernance.

TABLEAU 3. Synthèse des informations sur les mesures des variables

\begin{tabular}{lccllc}
\hline Construit & Dimensions & Items & $\begin{array}{l}\text { Coefficient } \\
\text { Alpha de } \\
\text { Cronbach }\end{array}$ & $\begin{array}{l}\text { Fiabilité } \\
(\mathrm{CR})\end{array}$ & $\begin{array}{l}\text { Variance } \\
\text { Moyenne } \\
\text { partagée (AVE) }\end{array}$ \\
\hline Gouvernance & 4 & 9 & 0.701 & 0.828 & 0.6 \\
\hline
\end{tabular}

(Tableau 4). Soit 0.828 pour le construit gouvernance. Les valeurs de CR et de l'AVE trouvées confirment que la validité convergente est adéquate pour la mesure sous examen (Haguma et al., 2019). La validité discriminante a été attesté en comparent les carrés des corrélations entre les variables et la variance moyenne partagée (Bagozzi et Yi, 2012). Le tableau ci-dessous présente les amples informations :

Les résultats du tableau ci haut montrent que les carrés de corrélation entre les variables sont inférieurs à la variance moyenne partagée y relative. Ces résultats indiquent que les construits sont différents les uns des autres et prouvent que l'échelle utilisée remplis les critères de validité discriminante.

Techniques de traitement des données. Les données ont été traitées en recourant au tri à plat, à l'analyse factorielle exploratoire et confirmatoire.

Le tri à plat nous a permis de décrire le comportement des dirigeants des PMEF de la ville de Bukavu face aux différentes facettes de la gouvernance. Ceci nous a permis d'appréhender l'importance que ces différents dirigeants ont accordée à la gouvernance pour la pérennité de leur PMEF dans un environnement en perpétuel mutation comme celui de la RD Congo. 
TABLEAU 4. Matrice de corrélation entre des construits et des dimensions

\begin{tabular}{|c|c|c|c|c|c|}
\hline Dimension & GOVEF & PARCIM & PERSON & PARTIP & SYSGES AVE \\
\hline PARCIM & 1 & & & & 0.51 \\
\hline PERSON & $\begin{array}{l}0.23 \ll \mathbf{0 . 0 5} » \\
(0.007)^{* *}\end{array}$ & 1 & & & 0.7 \\
\hline PARTIP & $\begin{array}{l}0.22 \ll \mathbf{0 . 0 5} » \\
(0.013)^{*}\end{array}$ & $\begin{array}{l}0.303 \ll \mathbf{0 . 1} » \\
(0.000)^{* *}\end{array}$ & 1 & & 0.683 \\
\hline SYSGES & $\begin{array}{l}0.21 \ll \mathbf{0 . 0 4} » \\
(0.013)^{*}\end{array}$ & $\begin{array}{l}0.284 \ll \mathbf{0 . 0 8} » \\
(0.001)^{* *}\end{array}$ & $\begin{array}{l}0.287 \ll \mathbf{0 . 0 8 2} » \\
(0.001)^{* *}\end{array}$ & 1 & 0.4 \\
\hline
\end{tabular}

Avec ( ) la probabilité ; « X » le carré de coefficient de corrélation ; * : significatif au seuil de 5\% et ** : significatif au seuil de $1 \%$

L'analyse factorielle en composante principale nous a permis de mesurer et d'extraire les dimensions de la gouvernance des PMEF(Stafford et Bodson, 2006). L'analyse a permis d'abord de vérifier que les données au construit admettaient l'analyse factorielle $(\mathrm{KMO}>0.50$ et le test de sphéricité de Bartlett avec chi-deux significatif $<0.05$ ) (Memmi, 2002). L'analyse a permis ensuite d'extraire les facteurs (valeur propre $>1$ ) via une démarche itérative en supprimant les items qui ne contribuent pas à expliquer les construits en utilisant la communalité $(>0.5)$ et les coefficients structurels $(>0.5)$ comme critères principaux (Carricano et Poujol, 2009). La rotation varimax a été utilisée pour maximiser la chance que chaque variable soit corrélée à une seule composante (Stafford et Bodson, 2006). Le processus de purification a été arrêté à lorsque un total de variance expliquée de $60 \%$ a été atteint (Haguma et al., 2019). L'analyse confirmatoire a été utilisée pour confirmer l'unidimensionnalité de chaque échelle en démontrant que les items et les dimensions identifiés par l'analyse exploratoire sont ceux qui caractérisent réellement le construit latent (Brookings et Bolton, 1988). L'analyse a permis de vérifier que tous les items identifiés exhibaient des coefficients structurels $>0,50$ (Nathalie, 2015). L'analyse a permis aussi de juger de la qualité d'ajustement des modèles de mesure et structurel en se référant aux indices préétablis (Khi deux non significatif, Khi-deux/df $<3$; RMSEA $<0.1$; CFI $>0.90$ ; IFI $>0.90$; NFI $>0.9$; GFI $>0.9$ et AGFI $>$ 0.9) (Hanana et Houfaidi, 2016). L'analyse a permis de confirmer que les échelles et leurs dimensions étaient fiables $(\alpha>0.70$ et $\mathrm{CR}>$ 0.70 ) et valides (AVE $>, 50$ et

$$
\rho v c i \geq \max \left(r_{n i, n j}^{2}\right)(\text { Lomax, 1982) }
$$

En outre, cinq indicateurs ont été utilisés pour attester la qualité d'ajustement du modèle estimé: Khi carré, $X^{2} / d f$, le RMSEA, le GFI et le CFI (Bagozzi et Yi, 2012). Le Khi-deux permettait de confirmer l'hypothèse d'égalité entre les matrices variance-covariance se rapportant à la fois à l'échantillon et au modèle soit $S-\varepsilon$ en recourant à sa statistique $x^{2}-(n-1) F_{\min }$. En suivant une distribution de $\chi^{2}-1 \frac{m(m+1)}{2} 1-k$, le bon ajustement étant prouvé pour une valeur de $\mathrm{X}^{2}$ faible et une signification associée élevée (Bagozzi et Yi, 2012). Nous avons aussi utilisé le ratio du Khi-deux/Df pour lequel des valeurs inférieures à 3 témoignaient d'un bon ajustement. Le Root Mean Square Error of Approximation (RMSEA) a été utilisé pour 
démontrer qu'il n'existe pas des différence entre la matrice de variance covariance du modèle sous analyse et celle de la population (Byrne, 2009). Ainsi, une valeur de RMSEA $<0.08$ était considérée comme preuve d'un bon ajustement pourvu qu'elle s'est trouvé dans l'intervalle de confiance de $90 \%$ (Hair et al., 2011). Le Comparative Fit Index (CFI) a été utilisé pour démontrer qu'il existait une différence statistique entre les matrices variance-covariances obtenues et la matrice variance-covariance du modèle de base (faibles corrélations entre les variables). Nous avons accepté le modèle pour une valeur supérieure ou égale à 0.9 (Bagozzi et Yi, 2012). Le Goodness of Fit Index (GFI) a servi d'identifier la proportion d'information expliquée par la matrice $\mathrm{S}$. Nous avons opté pour une valeur de GFI supérieure ou égale à 0.9 pour une bonne qualité du modèle (Lomax, 1982). En définitive, la fiabilité et la validité du construit latents ont été établies. La fiabilité a été testée en recourant à la fois aux coefficients á de Cronbach et au rho de Jöreskog (Brookings et Bolton, 1988). La validité de la variable latente a été obtenue à travers les validités convergente et discriminante. La variance moyenne extraite pour le construit et dimension a permis de tester la validité convergente. Cette dernière était confirmée lorsque les dimensions exhibaient des valeurs des variances moyennes extraites $(\mathrm{AVE})>, 50$ (Hair et al., 2011). Ces résultats signifiait que les items rattachés à chaque dimension expliquaient mieux cette dernière qu'ils ne le feraient pour d'autres dimensions. La comparaison de la variance moyenne extraite pour chaque dimension avec le carré de la corrélation simple entre les dimensions prises deux à deux, a permis de tester la validité discriminante (Bahia et Nantel, 2000).

\section{RÉSULTATS ET DISCUSSION}

Le Tri à plat. Les résultats du Tableau 5 cidessous indiquent que le score moyen de la gouvernance des PMEF de la ville de Bukavu était de 2.59 (39.75\%) relevant que les dirigeants de PMEF de la ville de Bukavu jugent leur niveau de gouvernance inférieur à la moyenne. Ces résultats corroborent notre deuxième hypothèse $(\mathrm{H} 2)$ suivant laquelle les PMEF de la ville de Bukavu dispose un niveau de la gouvernance légèrement supérieur à la moyenne. Ces résultats concordent avec ceux trouvés par Essama et al., 2015 et révèlent que les PMEF trouvent des difficultés pour devenir compétitives et accéder au financement formel (Hirigoyen, 2007). Ces difficultés sont dans la plupart des cas traduites

TABLEAU 5. Score moyen de la gouvernancedes PMEF de la ville de Bukavu, République Démocratique du Congo

\begin{tabular}{lccrrrrr}
\hline Items & $\mathrm{N}$ & Moyenne & Ecart type & $\mathrm{CV}$ & $\begin{array}{c}\text { Score en\% } \\
\text { moyen }\end{array}$ & Skewness & Kurtosis \\
\hline PARCI2 & 132 & 2,89 & 1.49 & 0.516 & 47.25 &, 169 & -1.455 \\
PARCI3 & 132 & 2,93 & 1,473 & 0.503 & 48.25 &, 032 & -1.414 \\
PERSO1 & 132 & 2,55 & 1,479 & 0.58 & 38.75 &, 549 & -1.124 \\
PERSO2 & 132 & 2,55 & 1,495 & 0.59 & 38.75 &, 537 & $-1,206$ \\
PARTI2 & 132 & 2,47 & 1,356 & 0.55 & 36.75 &, 625 &,- 859 \\
PPRRI3 & 132 & 2,51 & 1,438 & 0.573 & 37.75 &, 540 &,- 674 \\
SYSG2 & 132 & 2,56 & 1,366 & 0.53 & 39 &, 564 &,- 850 \\
SYSG3 & 132 & 2,39 & 1,335 & 0.56 & 32.25 &, 729 &,- 674 \\
SYSG4 & 132 & 2,46 & 1,378 & 0.56 & 36.5 &, 595 &,- 942 \\
GOUVEF & 132 & 2,59 & 0,77 & 0,297 & 39.75 &, 376 &,- 277 \\
\hline
\end{tabular}


par le faible degré de la bonne gouvernance adopté par les dirigeants des PME.

En outre, le coefficient de concentration (Kurtosis) était négatif (-0.277) (Tableau 5) a prouvé que les avis des dirigeants de la ville de Bukavu sur l'importance accordée à la gouvernance de leurs PMEF étaient partagés. Le coefficient de symétrie étant positif (Skewness $=0.376$ ) indique que les observations étaient dans l'ensemble concentrées vers les valeurs moins élevées. Ceci a révélé que les dirigeants des PMEF de la ville de Bukavu accordent une moindre importance à la gouvernance de leur PMEF. Dans le contexte de la ville de Bukavu, ces résultats pouvaient être justifiés par les difficultés que connaissent les dirigeants des PMEF pour utiliser les outils de gestion et de planification et partant mettre en place la bonne gouvernance. Ces résultats concordent avec ceux trouvés par Kamala, 2014 ; qui ont montré que les contraintes à la bonne gouvernance des PME limitaient leur capacité à répondre à leur mission et les rendaient moins compétitives et non crédibles pour accéder à des sources de financement formel (Tableau 5).

Le Tableau 5, montre que les dirigeants des PME de la ville de Bukavu accordaient une faible importance aux différentes facettes de la gouvernance. Le score d'importance accordé par les dirigeants aux différents éléments de la gouvernance a été en deçà de la moyenne. Tous les items ont disposé une dispersion plus élevée par rapport à la valeur centrale. La dispersion était arrivée jusqu'à 0.59 (soit $59 \%$ ) pour l'item « Rencontre avec les employés et conseil de famille» et 0.58 (soit 58\%) pour l'item « Communication avec les employés et cohésion famille».Cependant, pour tous les items de la gouvernance des PMEF de la ville de Bukavu, les coefficients de concentration (Kurtosis) étaient négatifs alors queles coefficients de symétrie (Skewness) étaient positifs. Ceux-ci avaient prouvé comparativement à la distribution normale que les observations ont été dans l'ensemble concentrées vers les valeurs les moins élevées. Ce qui indique que la quasitotalité des dirigeants des PMEF accordaient une moindre importance aux différentes facettes de la gouvernance et que les observations étaient fortement concentrées vers les valeurs moins élevées, mais les avis des dirigeants étaient partagés par rapport à l'importance qu'ils accordaient aux différentes facettesde la gouvernance. Toutefois, il a été constaté que les dirigeants des PMEF ont accordé un faible degré d'importance aux items relatifs au système de gestion. Le score moyen d'importance était arrivéjusqu'à $32.25 \%$ pour l'item «Mise en place des outils de contrôle et évaluation ». Ces résultats indiquent que les dirigeants des PMEF de la ville de Bukavu n'ont pas trouvé d'importance capitale à élaborer les outils efficaces de contrôle et d'évaluation. Dans le contexte de la ville de Bukavu, ces résultatsont été an partie justifiés par les techniques de gestion opaque, informelles et implicites applicables par les propriétaires dirigeants des PMEF qui conduisent à la dissimulation de l'information (Adolphe, 2018). Ceciaccroit le niveau de la perception de risque et le rationnement des PMEF par les institutions financières de notre environnement.

Relation entre la gouvernance et ses dimensions. Les résultats montrent que toutes les dimensions retenues de la gouvernance contribuent significativement à l'explication du construit (Tableau 6).

Les résultats montrent que chaque dimension est rattachée au construit latent avec $\mathrm{R}^{2}>0.2$ se référant à la variance expliquée est modérée et indique que le modèle est significatif (Hanana et Houfaidi, 2016). Toutes les dimesnions se rapportant à la gouvernance présentent des t- values supérieurs à 1.96. Ces résultats nuancent notre première hypothèse (H1) qui stipulent queles dimensions qui permettent de mesurer la gouvernance des PMEF de la ville de Bukavu en République 
TABLEAU 6. Gouvernance et ses dimensions dans les des PMEF de la ville de Bukavu en République Démocratique du Congo

\begin{tabular}{lcccc}
\hline Paramètres & Estimateur & Erreur standard & T Value & $\mathrm{R}^{2}$ \\
\hline Parcimonie $\leftarrow$ Gouvernance & 0.56 & 0.21 & 2.62 & 0.31 \\
Personnification $\leftarrow$ Gouvernance & 0.59 & 0.14 & 4.21 & 0.35 \\
Paricularisme $\leftarrow$ Gouvernance & 0.65 & 0.12 & 5.26 & 0.42 \\
Système de gestion $\leftarrow$ Gouvernance & 0.8 & 0.2 & 3.95 & 0.65 \\
\hline
\end{tabular}

Démocratique du Congo sont les parties prenantes, la parcimonie, la personnification, le particularisme et le système de gestion. Ils indiquent que les dimensions considérées permettent de saisir la gouvernance des PMEF de la ville de Bukavu. Ces résultats ont permis de corroborer les résultats relatifs aux dimensions de la gouvernance obtenus dans les études de (Carney, 2005;Cerrada et Jenssen, 2006; Essama et al., 2015).Ces résultats révèlent que les propriétaires dirigeants des PMEF de la ville de Bukavu apprécient leur gouvernance sur base de leur étendue de contrôle et indépendance dans la prise de décision, la bonne combinaison de la relation propriété- famille - entreprise et le maintien de la réputation inter générationnelle de la famille. Ces résultats sont justifiés dans le cadre de la ville de Bukavu par le fait que les propriétaires dirigeants des PMEF conçoivent leurs entreprises comme source d'emploi pour les familiers, incubatrices des compétences et des richesses pour sauvegarder l'image et le bien être de la famille au fil de génération.

\section{CONCLUSIONS, LES IMPLICATIONS, LES LIMITES ET RECHERCHES FUTURES}

Les résultats de l'analyse de la gouvernance des PMEF de la ville de Bukavu en RDC confirment que la gouvernance est un construit à neuf items regroupés en quatre dimensions (la parcimonie, le particularisme, la personnification et le système de gestion). Ils révèlent aussi que les dirigeants des PMEF accordent un degré faible d'importance aux différentes facettes de la gouvernance et que leurs avis sont partagés sur l'importance accordée à la gouvernance.

Le faible score d'importance accordée à la gouvernancepar les dirigeants des PMEF de la ville de Bukavu rappelle que les efforts doivent être fournis pour inciter la bonne gouvernance au sein des PME.Cet aspect permettra aux PME d'accroitre leur échelle d'activité, à devenir compétitives et assurer aisément leur rôle de créateur d'emploi. Les avis des dirigeants par rapport à l'importance des différentes facettes de la gouvernance confirment que la gouvernance est le central des difficultés que connaissent les PMEF.

Bien que cette étude aboutisse à des résultats probants sur le plan scientifique, elle souffre toutefois de trois limites principales. Premièrement, l'échelle utilisée est explorée sur un échantillon des PME familiales. Une étude future mesurant la gouvernance des PME en tenant compte des spécificités des toutes les PME permettrait de comparer les résultats et identifier le différentiel de niveau de gouvernance entre les PMEF et non familiales. Deuxièmement, l'étude n'a porté que sur un seul site ayant une caractéristique particulière : la ville. Ce qui pourrait limiter la portée et la généralisation de résultats. Une recherche ciblant à la fois les PME des milieux ruraux et urbains permettrait de compléter les résultats de cette recherche. Une étude rétrospective portant sur la gouvernance, la réussite de la succession et performance des PMEF est aussi envisagée. Elle permettrait d'étudier la 
capacité de la bonne gouvernance à influencer la performance intergénérationnelle à travers la réussite de la succession. Ceci aidera à porter une attention sur le niveau de la perception de la succession des PMEF et identifier les capacités de la bonne gouvernance des dirigeants sur la préparation de leurs successeurs.

\section{BIBLIOGRAPHIE}

Adolphe, M. 2018. Mémoire DEA: Choix de financement et performance des PME en RDC. Bukavu: Université Catholique de Bukavu.

Aliyua, N.S., Jamila, C.Z. et Mohameda, E.R. 2014. The mediating role of management control system in the relationship between corporate governance and the performance of bailed-out banks in Nigeria. Procedia Social and Behavioral Sciences 164:613 620.

André, P., Khemakhem, H., et Sakka, O. 2006. Interdépendance des mécanismes de gouvernance : 27ème Congrès de l'Association Francophone de Comptabilité.

Arrègle, J.-L. et Mari, E. I. 2010. Avantages ou désavantages des entreprises familiales: Principaux résultats des recherches et Perspectives. Revue Française de gestion 1(200):87 - 109.

Assia, H. 2016. Gouvernance et Performance des PME dans les Pays en Transition.

Attouch, H. et Amria, E. F. 2016. Gouvernance des entreprises familiales : Un essai théorique sur la spécificité . Révue D'Etudes en Management et Finance D'Organisation 2:1- 18.

Bach, L., Cottet, S. et Monnet, M. 2018. Changement de gouvernance et performance des entreprises. France : Institut de Politique Publique 19.

Bagozzi, R. et Yi, E.Y. 2012. Specification, Evaluation and Interpretation of Structural Equation Models. Journal of Academy of Marketing Science 40:8-34.
Bahia, K. et Nantel, E.J. 2000. A reliable and valid measurement scale for the perceived service quality of banks. International Journal of Bank Marketing 18(2):84-91.

Banque Mondiale. 2014. Rapport sur le doing business. ND: Banque Mondiale.

Bauweraerts, J. et Colot, E.O. 2015. Performance des entreprises familiales et choix d'une technique de transmission: quelle influence? Management et Avenir 5(79): 157 - 175.

Bazika, J.-C. 2014. L'entreprise familiale: Définition et fonctionnalité dans une perspective Africaine. Ngouabi: CERAPE.

Belkebir, B., Daanoune, R., et Mouallim, I. 2018. Analyse de l'Impact de la Gouvernance sur la Performance: Cas des Banques Marocaines. International Journal of Innovation and Applied Studies 756767.

Bernstein, R. S., Buse, K., et Bilimoria, e. D. 2016. Revisiting Agency and Stewardship Theories: Perspectives From Nonprofit Board Chairs and CEOs. SIAS Faculty Publications 551:1-21.

Bocquet, R. et Mothe, E.C. 2008. Gouvernance et performance des pôles de PME. Revue Française de Gestion 10(190):101-122.

Brookings, J. et Bolton, E.B. 1988. Confirmatory factor analysis of the interpersonal support evaluation list. American Journal of Community Psychology 16(1):137- 147.

Brouard, F. et Divito, E.J. 2008, Octobre 28 31. Identification des mécanismes de gouvernance applicables aux PME. Congrès International Francophone en Entrepreneuriat et PME: 1-19.

Bughin, C., Colot, O. et Finet, E.A. 2010. Entreprises familiales et gouvernance cognitive: quelle transmission. Management et avenir 7(37):14-33.

Byrne, B. 2009. Structural Equation Modeling With LISREL, PRELIS, and SIMPLIS: Basic Concepts, Applications, and Programming. Psychology Press Taylor and Francis, ND. 
Caillie, D. V. et Onana, E. A. 2012. L'influence de la gouvernance familiale sur la transmission de la PME familiale au Sénégal : une modélisation contingente: pp. 1-23.

Carney, M. 2005. Corporate governance and competitive advantage in family controlled firms. Entrepreneurship Theory and Practice 29(3):249 -265.

Carricano, M. et Poujol, F. 2009. Analyse de données avec SPSS. France: Pearson ducation.

Cerrada, K. et Janssen, F. 2006. De l'applicabilité, des spécificités et de l'utilité d'un code de gouvernance d'entreprise pour les PMe et les TPE : le cas de la Belgique. Revue Internationale P.M.E.: pp. 163-193.

Charreaux, G. 1998. Le système de gouvernance des entreprises. Université de Bourgogne: Pôle d'Économie et de Gestion, 21000 Dijon.

Charreaux, G. 2008. A la recherche du lien perdu entre caractéristiques des dirigeants et performance de la firme: gouvernance et latitude managériale.

Charreaux, G. et Schatt, E.A. 2005. La recherche française en gouvernance d'entreprise : un panorama. Cahier $d u$ FARGO $n^{\circ}$ 1050901: 1-37.

Claessens, S., Djankov, S. et Lang, E.L. 2000. The separation of ownership and control in East asian corporations. Journal of Financial Economics 1-2(58):81 -112.

Colot, O. et Mpasinas, E.A. 2007. Influence des caractéristiques propres aux entreprises familiales et à leurs dirigeants sur la performance: une étude basée sur les entreprises Belges. Journal of Small Business ans Entrepreneurship 20(1):69-86.

Comblé, K. et Colot, E.O. 2006. L'entreprise familiale: concept et importance en Belgique. Reflets et Perspectives de la vie Economique XLV(2): 91 - 108.

Davis, P. et Pett, E.T. 2000. Governance and goal formation among family businesss: A ressource dependency perspective. The
International Journal of ENtrepreneurship and Innovation 1(3):137 -149.

Djimnadjingar, R. 2012. Gouvernance et performance des PME familiales au Tchad. Lille : Lille Economie management.

Essama, P., Kamga, A. F. et Zama-Akono, C. 2015. Gouvernance et performance des Institutions de Micro Finances au Cameroun. La Microfinance en Afrique: Les Defis des Exclus: 170-188.

Haguma, B.M., Balemba, E.K. et Bitakuya, E.W. 2019. Relation entre microfinance et la performance perçue des PME: Rôles médiateur et modérateur de l'opportunité entrepreneuriale et de la prise de risque. Finance Contrôle et Stratégie 22(4):1-44.

Hair, Ringle et Sarstedt, E.M. 2011. PLS-SEM: indeed a silver Bullet :Theory and Practice. Journal of Marketing 19(2):139-151.

Hanana, E.B. et Houfaidi, E.S. 2016. Satisfaction du personnel: validation d'une échelle de mesure dans le contexte des administrations publiques Marocaines. International Journal of Innovation and Applied Studies 18(4):1056-1073.

Hirigoyen, G. 2007. Biais comportementaux et mécanismes régulateurs dans la succession de l'entreprise familiale. Revue Française de Gouvernance d'Entreprise 1:67-90.

Hirigoyen, G. 2009. Concilier finance et management dans les entreprises familiales. Lavoisier, Revue Française de Gestion 198199(8): 393-411.

Kamala, C.K. 2014. PME et développement: atouts, contraintes institutionnelles et perspectives dans le contexte de la RDC. Bukavu Journal of Economies and Social Sciences: 1-20.

Kamba-Kibatshi, M. 2016. L'influence des petites et moyennes entreprises au développement économique de la République Démocratique du Congo. Nierównoxci Spo3eczne a Wzrost Gospodarczy: 478-505.

Kenyon-Rouvinez, D. et Ward, E.J. 2004. Les entreprises familiales. Paris: Presse universitaire de France. 
Khaldi, A.M. 2014. Impact des mécanismes de gouvernance sur la création.

Kolsi, M. et Ghorbel, H. 2011. Effet des mécanismes de gouvernance sur la performance financière et boursière : Cas des entreprises canadiennes.

Krafft, J., Qu, Y. et Ravix, J.-L. 2010. Gouvernance d'entreprise et performances sectorielle:une réevaluation de la fiabilité des scores et des mesures de bonne gouvenance.

Landier, A., Sraer, D. et Thesmar, E.D. 2004. Going for broke. ND: New Centry Financial Corporation.

Larue, D. 2005. La gestion par les compétences, un outil au service du projet stratégique. Paris: CNAM.

Lomax, R. 1982. Methods and designs: A guide to Lisrel-type structural equation modeling - Behavior Research Methods and instrumentation 14(1):1- 8 .

Mbenda, S. P., et Sebigunda, e. E. 2015. Efficacité des mécanismes de gouvernance des PME : Une évaluation empirique en contexte camerounais. Revue Internationale P.M.E. 28(1):57-85.

Memmi, D.E. 2002. Ananlyse factorielle Neuronale pour Documents Textuels, les cahiers du laboratoire Leibniz. Leibniz: GRENOBLE.

Mendy, M. et Diop, E.A. 2018. Profil du dirigeant, choix des mécanismes de gouvernance et performancedes entreprises familiales africaines: une analyse à partir des données sénégalaises. Revue Africaine de Management 3(1):69-92.

Morck, R. et Yeung, E.B. 2003. Agency Problems in large family business group. Entrepreneurship Théory and Practice 27(4):367-382.

Nathalie, G. 2015. Compétences relationnelles et multibancarité: quels impacts sur le financement de la PME: Une application aux
PME et PMI aéronautiques . XXIVème Conférence Internationale de Management Stratégique (p. ND). Paris: Association Internationale de Management Stratégique. Ndalo, D.T. 2010. Analyse des causes de contre- Performance de PME congolaise. Cas de PME de la commune de Lemba. Kinshasa, RDC.

Ngongang, D. 2005. Pratiques comptables, système d'information et Performance des PME Camérounaises. Revue des Sciences de Gestion 6(216):59 - 70.

Ngongang, D. 2014. Analyse de la compétence des Propriétaires - dirigeants des PME du Nord Caméroun. Revue Congolaise de Gestion 19(1):9-42.

Ntambaka, G.A. 2015. Les déterminants d'accès des PME au crédit des institutios financières. Bukavu: UCB.

O'grady, J. 2002. Recent corporate failures Key lessons from the private sector. Conference on Corporate Governance in the Public Sector, Canberra. p. ND.

Premont, A. 2016. Étude des liens entre les caractéristiques du conseil d'administration et la performance de l'entreprise .

Ramadani, V., Springer, C. et Hoy, E. 2015. Context and uniqueness of family businesse. In: Family businesses in transition economies: 9 - 37.

Stafford, J. et Bodson, E.P. 2006. L'analyse multivariéé avec SPSS. Quebec: Presse de l'université du Quebec.

St-Pierre, J. et Cadieux, E.L. 2011. La conception de la performance: Quels liens avec le profil entrepreneurial des propriétaires dirigeants de PME. Revue de l'Entrepreneuriat 1(10):33-52.

Yassine, M. 2013. Le développement des PME et la bonne gouvernance «Cas filiale transcanal». Ouest SPA, Algérie: Unité II Relizane. 